\title{
Tendances mondiales et incidences sur les pratiques forestières au Canada ${ }^{1}$
}

\author{
par Maureen $\mathrm{O}^{\prime} \mathrm{Neil}^{2}$
}

Comme l'ont mis clairement en évidence mes collègues, les experts-forestiers et les autres professionnels des ressources naturelles doivent faire face à une multiplicité de nouveaux défis. Dans le monde d'aujourd'hui, les collectivités demandent que la gestion de leurs territoires boisés ne se fasse pas uniquement en fonction de la production ligneuse mais débouche sur un large éventail de biens et services.

Les demandes sont nombreuses et souvent concurrentes. Nous avons beaucoup parlé - et ils sont légion ceux qui ne font que parler - de développement durable. Mais puisque nous avons appris de plus en plus de quoi il est vraiment question — ou de ce qu'il devrait être — nous soulignons la nécessité de réaliser un développement durable et équitable à la fois. Ces deux qualités sont indissolubles comme des jumelles siamoises.

Le thème de cette conférence me donne l'occasion d'aller au coeur du sujet. Une des raisons pour lesquelles j'éprouve un grand plaisir à être parmi vous aujourd'hui est que cette réunion nous donne la chance d'explorer ensemble la nature de ces liens mondiaux et locaux et, plus encore, d'envisager de quelle manière nous pourrions créer des partenariats et des réseaux qui s'affrontent de manière plus efficace aux questions économiques et sociales qui revêtent une importance vitale dès lors que nous tentons de gérer et de sauvegarder nos forêts. Nous avons dit durable et équitable.

Mais d'abord, permettez-mois d'expliquer pourquoi cette question me touche d'aussi près. Un enfant qui naît aujourd'hui dans notre ville d'Ottawa ou à New York, Rome ou Tokyo, consommera, gaspillera et polluera au cours de son existence plus que ne le feront cinquante enfants d'un pays en développement. Disons la vérité - à notre époque, ces derniers n'en auront même pas la chance! Et par un coup du sort cruel, ceux qui consomment moins porteront le poids du dommage écologique qui s'ensuit.

Cette observation est contenue dans le plus récent Rapport annuel des Nations Unies sur le développement humain publié il y a quelques semaines. C'est le même rapport qui place encore une fois le Canada au premier rang des nations dont les citoyens, dans l'ensemble, jouissent d'une qualité de vie sans égal dans le monde. En tant que Canadienne, je suis fière de ce constat. Mais il nous faut poser la question : À quel prix?

À prix fort, lorsque des pays industrialisés modernes comme le nôtre sont des consommateurs dominants. Les populations des pays les plus pauvres du monde paient - par comparaison à des gens comme nous - le prix le plus élevé du fait de la pollution et la dégradation des sols, des rivières, des océans et des forêts dont elles dépendent pour leur subsistance.

\footnotetext{
${ }^{1}$ Communication aux délégués de la $90^{\mathbb{e}}$ Assemblée générale annuelle de l'Institut forestier du Canada

${ }^{2}$ Présidente, Centre de recherches pour le développement international
}

L'époque à laquelle nous vivons a connu une croissance sans précédent dans l'histoire, à la fois par l'ampleur et la diversité de ses perspectives économiques. Il est indéniable que de nos jours plus de gens sont mieux alimentés, mieux logés et bien portants qu'à toute autre époque. À l'approche du nouveau millénaire, les niveaux de vie ont progressé au point que des centaines de

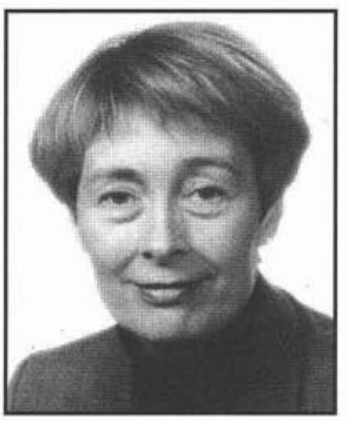
millions de personnes jouissent d'une qualité de vie inimaginable pour leurs grand-parents ou aïeuls il y a un siècle à peine.

Ce malgré, nous traversons une période de l'histoire chargée de menaces. Les modèles mondiaux de consommation approfondissent les inégalités.

Comme le fait remarquer Amartya Sen, économiste avisé:

« Il est impérieux que le concept étroit de développement économique soit remplacé par la notion plus ample de développement social. Ce qui compte en dernier ressort sont les conditions de vie que les gens peuvent - ou ne peuvent pas - mener. »

En d'autres mots, le succès ou l'échec du développement économique ne peut être simplement jugé en termes de revenu et de rendement.

Près de 20 pour cent des habitants de la planète vivent dans des pays à revenu élevé comme le nôtre et consomment neuf dixièmes de tous les biens et services produits sur le globe. $\mathrm{Ce}$ cinquième de la population nantie (vous et moi) contribue à la consommation de 85 pour cent environ du papier produit dans le monde. La consommation mondiale de bois s'est accrue de 40 pour cent depuis le début des années 1970, confirme le Rapport sur le développement humain publié le mois dernier par les Nations Unies.

Mais il ne faut pas omettre de prendre en compte un autre important facteur. Depuis 1970, sur la planète les zones boisées du monde par habitant ont diminué de plus d'un tiers (passant de 11,4 kilomètres carrés par mille habitants à 7,3 kilomètres carrés aujourd'hui). Et, à la différence de la génération ou des deux générations qui nous ont précédé, les pertes se concentrent dans les pays du Sud et non dans les pays industrialisés.

Au cours des dix dernières années, la superficie d'une forêt tropicale équivalente à celle de la Colombie-Britannique et de l'Alberta réunies a irrémédiablement disparu.

Nous sommes tous conscients qu'une dévastation à cette échelle a de graves répercussions par la perte de la biodiversité, le changement des microclimats et pire encore.

Dans de nombreuses régions en développement, les villageois pauvres qui peuvent tirer au moins la moitié de leurs aliments des territoires boisés n'ont jamais connu la famine. Cette capacité est aujourd'hui en voie de se perdre.

Aux Philippines, la moitié des forêts du pays ont succombé à l'abattage commercial sous la dictature Marcos. Dix-huit 
millions d'habitants ont été laissés sans ressources. Les conséquences sociales d'un tel bouleversement sont démesurées.

Il n'y a aucune échelle de Richter qui puisse mesurer des perturbations terrestres d'une telle ampleur.

Quelques-uns croient que les pays pauvres d'Afrique, d'Asie et d'Amérique latine devraient restreindre leur niveau de consommation afin de limiter les dommages environnementaux. Facile à dire. Mais cela signifierait également prolonger un état de privation déjà scandaleux en soi.

Pris dans leur ensemble, les pays en développement du Sud font face à un défi stratégique de taille. Doivent-ils reproduire les processus d'industrialisation et de croissance de pays comme ceux qu'a suivi le nôtre au cours des cinquante dernières années - et subir une catastrophe écologique sous prétexte d'un rattrapage et de l'avènement d'un semblant de justice économique?

Ou pourront-ils progresser en adoptant une approche respectueuse de l'environnement, selon une évolution capable de préserver les ressources naturelles et donc moins défavorables aux pauvres, créatrice d'emploi et de logement, donnant accès aux services sociaux de base que réclame une existence vécue dans la dignité.

Il n'y a pas de doute que celui-ci est le chemin de la justice et celui qui rend justice. C'est aussi la voie du sens commun. En l'empruntant, les pays en développement peuvent accélérer l'accès de leurs populations à une vie meilleure tout en minimisant le coût écologique. Ils peuvent également être encouragés à se tourner vers des technologies accessibles plus propres et moins énergivores.

Par exemple, en Afrique - et plus précisément en Tanzanie - au début des années 1990, le CRDI a entrepris un projet visant à réduire la dépendance des producteurs de contreplaqué local vis-à-vis des matières importées. Les chercheurs sur le terrain ont acquis de nouvelles connaissances qui leur ont permis de mettre au point des adhésifs et des agents de conservation simples et fiables extraits de ressources locales.

Le CRDI a été créé par le gouvernement du Canada pour aider les pays les plus démunis à entrer dans l'ère moderne de la science et de la technologie afin d'améliorer les conditions de vie de leurs populations. Lester B. Pearson, notre ancien Premier ministre et premier président du Conseil des gouverneurs du CRDI, déclarait il y a trente ans que nous devons également aider ces pays à évoluer en ayant soin d'éviter les menaces susceptibles de mettre en péril leurs valeurs et traditions confirmées, leur milieu de vie et leur culture, sous la poussée d'un progrès technologique incontrôlé. Aujourd'hui, avec tout le recul nécessaire, nous sommes peut-être mieux en mesure de comprendre que cela n'était qu'un cri d'espoir — pour les bonnes ou les mauvaises raisons peu importe. Il y avait toutefois, au coeur de la déclaration de Pearson, une exhortation qui est demeurée une de nos préoccupations constantes, à savoir le respect que nous devons avoir à l'égard de tous ceux que nous avons la chance de côtoyer.

De nos jours, il apparaît urgent de répondre aux besoins et, bien sûr, d'élever le niveau de consommation d'un milliard de pauvres qui constituent le quart de l'humanité laissé pour compte, abattu comme la forêt, privé d'espoir.

Nous qui sommes dans cette salle, avons raison de nous sentir interpellés et avons l'obligation de réfléchir à ces réalités d'une manière plus grave afin de commencer à répondre à leurs besoins tout en nous dirigeant nous-mêmes vers des modèles de consommation plus durables.
Nous préconisons donc un développement durable mais équitable. Des modèles de croissance qui réduisent les niveaux actuels de destruction environnementale. Des politiques qui rendent plus facile - mais aussi économiquement attrayant - de consacrer les efforts de la nouvelle recherche et de l'investissement à la régénération des ressources renouvelables : le bois, l'eau, les sols, les poissons.

Mais rien de tout cela ne peut fonctionner si nous ne saurons établir des liens de coopération plus étroite et des réseaux mondiaux plus efficaces parmi les sociétés nanties et les pays en développement, au moment même où tous les pays de l'OCDE confondus y consacrent en chiffres réels seulement deux tiers de ce qu'ils dépensaient il y a quinze ans.

J'ai cité Lester Pearson il y a quelques instants. Je le ferai encore.

Il fit valoir un autre point qui revêt de nos jours une grande importance, à savoir que l'esprit dans le quel la main est tendue et l'assistance prêtée est tout aussi importante que l'aide elle-même. En d'autres mots, qu'il devrait y avoir du côté de celui qui reçoit un sentiment de participation et de réalisation de soi, d'engagement et de partenariat. Et que nous ne pouvons ni ne devrions attendre la gratitude des pays pauvres pour « les miettes ou même le pain qui tombe de la table du riche », pour reprendre ses mots et ceux de la parabole.

Une génération plus tard, au moment où sur la planète nous approchons des six milliards d'habitants je me demande : Comment venir en aide aux populations du tiers monde sur la planète par des programmes qui se justifient de leur propre point de vue?

L'organisme que j'ai l'honneur de diriger — le CRDI n'a pas de mandat particulier pour l'exécution de programmes d'aide extérieure, tels qu'on les comprend généralement. Notre action, notre mission a un caractère beaucoup plus général : l'aide à la recherche pour le développement. En bref, l'argent du contribuable canadien aide les gens d'autres pays à créer la capacité d'édifier par soi-même un monde plus juste.

Nous ne saurions, en fin de compte, donner une meilleure définition du développement durable et équitable.

Le CRDI a pour devise «S'affranchir par le savoir ». Et je puis vous dire - en tant que présidente du CRDI - que rien n'est plus inspirant de monter jusqu'à mon bureau chaque matin dans l'ascenseur entourée de scientifiques de classe internationale et d'autres chercheurs qui vivent cette réalité dans notre ville ou dans un de 142 pays en développement où le Centre a oeuvré au cours des trente dernières années.

Riche d'une expérience de presque trente ans, nous avons tissé des centaines de réseaux reliant des dizaines de milliers de chercheurs, scientifiques et auteurs de politiques. Nous avons appris, ne serait-ce que le débat public, le pluralisme et la compétition des idées - toutes composantes caractéristiques de la démocratie - dépendent du savoir qui souvent découle de la recherche bien orientée. Dans ce cas, les modalités de la recherche se révèlent tout aussi importantes que son contenu.

Plus simplement, tout ce qui ne témoigne pas d'une démarche démocratique - participative, plurielle, conduisant à des résultats aisément partagés — est une perte de temps. Participation, inclusion, accessibilité des résultats, telle est la force des réseaux mis en place.

Malheureusement, dans un grand nombre de sociétés, les réalités politiques dominantes ont exclu et marginalisé une grande fraction de la population en lui déniant ce droit à la participation : les peuples autochtones, les femmes, et d'autres catégories encore. 
Il est essentiel de les faire participer au débat et de les associer aux décisions.

Dans les régions en développement où l'extraction des ressources est orientée surtout vers l'exportation, ces groupes sont les principaux dépositaires des connaissances. Aussi, ils méritent d'être respectés pour ce qu'ils sont : les acteurs clés de tout débat informé sur les questions sociales et économiques et sur les évolutions susceptibles de modifier en profondeur le seul monde qu'ils auront la chance de connaître.

Sans cet engagement, sans participation communautaire aux solutions proposées pour une gestion des ressources avisée, les politiques prônant le développement durable sont destinées à échouer.

Sur le plan international, ce maillage donne à tous ces acteurs l'occasion d'échanger des expériences et des stratégies avec les homologues d'autres pays appartenant même à des cultures différentes et de nouer des solidarités avec leurs semblables confrontés à des défis analogues dans d'autres pays. Il se crée ainsi les conditions qui rendront possible leur propre inclusion dans les processus de prise de décision politique.

Au CRDI, une société d'État créée par le Parlement du Canada, nous croyons qu'un maillage comme celui que je décris est l'outil essentiel de l'édification d'un monde plus juste. Un monde qui, tout en prenant son essor, demeure enraciné dans un modèle de développement équitable et durable à la fois. Cela dit, $\mathrm{j}$ 'aimerais partager avec vous quelques exemples de notre action dans le domaine de la foresterie. Et aussi dans celui du secteur minier, qui a avec ce dernier un grand nombre de points communs.

Nous venons à peine de commencer un projet quinquennal de recherche novateur et axé sur la prospection minière et le développement en Amérique latine et Caraỉbes dans le but de rendre ces activités plus viables d'un point de vue environnemental.

Le projet vise à réaliser cet objectif en réduisant la distance entre les chercheurs et les utilisateurs de la recherche et en rendant les priorités de recherche plus proches des politiques d'intérêt public qui touchent toutes les parties en cause : collectivités locales, instances gouvernementales, intérêts d'entreprise et ONG. Il vise en outre à permettre une meilleure compréhension des aspects positifs et négatifs d'un développement attentif à l'environnement, et notamment des impacts positifs et négatifs - sur les cultures autochtones afin d'associer tous les intervenants au processus d'investigation et proposer le cadre juridique, réglementaire et politique qui convient le mieux.

Les Canadiens - et donc nous tous dans cette salle - savent pertinemment que si l'activité minière ou l'exploitation de la forêt peut amener des élans d'activité économique bienfaisante, elle peut laisser les traces de la dévastation dans son sillage. Les conséquences négatives peuvent aller de la pollution aux accidents du travail dans une proportion alarmante et à la dislocation de la collectivité. Nous reconnaissons tous que là où certaines activités menacent de laisser des populations entières plus démunies qu'auparavant se pose la question de la responsabilité morale.

Le rêve de tout prospecteur - et de tout dirigeant d'activité minière - est de trouver le filon qui le rendra prospère. Nous croyons que les responsabilités s'enracinent dans nos propres rêves. Qu'il s'agisse d'activités minières ou d'activités forestières, ces responsabilités commencent par la protection des intérêts collectifs au travers d'une gestion responsable de l'écosystème.

L'inaction qui n'est rien d'autre que l'incapacité de faire son devoir, a été un obstacle majeur à la santé d'un grand nombre de sociétés des régions en développement. L'expérience du Canada, pays fragile s'il en est du point de vue écologique, est révélatrice à cet égard.

Nous, Canadiens - avec notre immense frontière, une population autochtone importante et un passé distinctif d'exploitation des ressources naturelles - avons appris au moins une chose. Une approche durable et juste du développement économique dicte une conduite qui doit être à la fois démocratique et socialement solidaire.

Plus près de chez nous, et voilà qui explique ma présence parmi vous aujourd'hui, le CRDI a eu le plaisir d'héberger dès 1995 au siège social le Secrétariat du Réseau international de forêts modèles. Le terme « forêt modèle » évoque des images de sanctuaires immaculés éloignés de l'intrusion des tronçonneuses et des tracteurs.

En réalité, ce sont des gens qui font les forêts modèles et qui se distinguent par la manière dont ils agissent et savent établir une interaction avec l'écosystème forestier et les nombreuses ressources connexes. C'est cette relation qui nous vient à l'esprit lorsque nous employons le mot "modèle ». Les forêts modèles sont l'occasion de créer des partenariats communautaires et d'apprendre à prendre les décisions ensemble et à travailler de concert pour une gestion durable de la forêt.

Les Forêts modèles sont nées au Canada en 1992 à titre d'expérience pilote innovatrice. Sous le guide du Service canadien des forêts du gouvernement fédéral, l'initiative constitue à bien des égards une réalisation canadienne exemplaire. Et une expérience qui aujourd'hui se propage à l'échelle internationale.

L'expérience canadienne est la pierre angulaire du Réseau international des forêts modèles résidant au CRDI. En coopération avec nos partenaires nationaux, nous travaillons pour étendre ces réseaux sur tous les continents. On dénombre aujourd'hui des forêts modèles au Mexique, en Russie, au Japon, au Chili et aux États-Unis. L'Argentine, la Chine et d'autres pays devraient bientôt se joindre au réseau.

Je m'arrêterai ici. Mais avant de vous quitter, j'aimerais raconter une anecdote qui illustre bien le message que j'essaie de vous transmettre aujourd'hui.

Il y a quelques années, il m'a été donné de rencontrer un ami qui travaillait en Amérique centrale comme missionnaire canadien. C'était durant la période sanglante constellée de violations des droits de la personne et des dictatures qui se sont alternées dans cette région vers la fin des années 1980.Durant une visite au pays, il me dit qu'en traversant le Canada, il avait été étonné et troublé de constater un sentiment d'aliénation et de résignation universel.

S'il se rendait dans l'Est ou dans l'Ouest, les gens imputaient la responsabilité de leur situation au Canada central, en déclarant leur impuissance à modifier les choses. À Toronto, on rejetait le blâme sur Ottawa. Il venait ici et c'est Toronto que l'on tenait pour responsable de tous nos maux.

Il établissait la comparaison entre notre état d'esprit et celui des populations qu'il côtoyait au Salvador. Elles étaient entourées par les horreurs de la guerre. Elles vivaient sous une menace constante. Ceux qui les gouvernaient ne les représentaient en rien. 
Pourtant, en quelque sorte, ces gens se sentaient intimement solidaires de la vie civile de leur pays. Ils croyaient encore pouvoir marquer une différence et améliorer les choses. Ils n'étaient pas découragés. Ils n'étaient pas désespérés.

Je crois que nous devrions tirer vigueur et inspiration du comportement des citoyens du Salvador dans une des époques sombres de leur histoire.

Nous devrions ressentir l'obligation d'accorder notre soutien aux éléments de la société qui, bien que divisés, peuvent encore indiquer ce que travailler ensemble veut dire pour améliorer le sort de chacun, malgré un manque de moyens qui ne peut leur être imputable.

Plus encore. Il serait opportun que nous ressentions plus que la simple obligation d'aider les pays les moins nantis à gérer leurs ressources de manière plus équitable et durable. En les aidant à adopter des instruments adaptés à réaliser par eux-mêmes ces objectifs.

Responsabiliser les populations dans des régions des antipodes pour qu'elles deviennent capables d'agir dans leur propre intérêt et de contribuer à l'édification de sociétés plus justes, voilà qui peut sembler à certains une entreprise ésotérique.

Et pourtant.

Je parie qu'il s'agit de la science la plus inexacte qui soit dans le monde d'aujourd'hui. Mais pour ceux qui choisissent d'y investir et de s'investir, elle peut se révéler à terme la plus profitable de toutes.

Sachons du moins en prendre le risque. 